\title{
On the Monotone Column Permanent conjecture
}

\author{
James Haglund $^{1}$ and Mirkó Visontai ${ }^{1}$ \\ ${ }^{1}$ Department of Mathematics, University of Pennsylvania, 209 South 33rd Street, Philadelphia, PA 19104
}

\begin{abstract}
We discuss some recent progress on the Monotone Column Permanent (MCP) conjecture. We use a general method for proving that a univariate polynomial has real roots only, namely by showing that a corresponding multivariate polynomial is stable. Recent connections between stability of polynomials and the strong Rayleigh property revealed by Brändén allows for a computationally feasible check of stability for multi-affine polynomials. Using this method we obtain a simpler proof for the $n=3$ case of the MCP conjecture, and a new proof for the $n=4$ case. We also show a multivariate version of the stability of Eulerian polynomials for $n \leq 5$ which arises as a special case of the multivariate MCP conjecture.
\end{abstract}

Résumé. Nous présentons des développements récents concernant la conjecture Monotone Column Permanent (MCP). Nous utilisons une méthode générale pour prouver qu'un polynôme univarié a uniquement des racines réelles, c'està-dire que nous prouvons qu'un polynôme correspondant a plusieurs variables est stable. Les nouveaux liens, établis par Brändén, entre la stabilité des polynômes et la propriété forte de Rayleigh, permettent de vérifier facilement la stabilité de polynômes multi-affines. En utilisant cette méthode nous obtenons une preuve plus simple pour la conjecture MCP pour le cas $n=3$, et la première preuve pour le cas $n=4$. Nous présentons également une version multivarée de stabilité des polynômes d'Euler pour le cas $n \leq 5$, qui apparaît comme un cas spécial de la conjecture MCP multivarée.

Keywords: Monotone column, permanent, polynomials with real roots only, stability, strong Rayleigh property 


\section{Introduction}

We discuss some recent progress on the Monotone Column Permanent (MCP) conjecture of Haglund, Ono and Wagner (HOW99; Hag00).

The Monotone Column Permanent (MCP) conjecture Let $A$ be an $n \times n$ matrix with real entries weakly increasing down columns, i.e., $a_{i, j} \leq a_{i+1, j}$ for $i=1, \ldots, n-1, j=1, \ldots, n$. Then, the polynomial $p(z)=\operatorname{per}(B)$, the permanent of matrix $B$ with $b_{i, j}=a_{i, j}+z$ has only real zeros.

The conjecture was proven for some special cases in (HOW99), but the general case was left open for $n>3$ and the proof for the $n=3$ case was rather lengthy (May). In this paper we give a new proof for the $n=3$ case and prove the $n=4$ case. We also prove a special case for $n=5$ and conjecture a multivariate version of the stability of Eulerian polynomials.

\subsection{Real rootedness and stability}

To prove real rootedness of a polynomial $f$, i.e., that all roots of $f$ are all real, we will use a method of showing that a multivariate generalization of $f$ is stable. Similar ideas have been applied before in different contexts, e.g., the multivariate Heilmann-Lieb and Lee-Young theorems (HL72; Sok05), and recently remarkable results were proved concerning reality of roots, using stable polynomials in (BB08).

We start our discussion with some necessary definitions first.

Stability We call a polynomial $f \in \mathbb{R}\left[z_{1}, \ldots, z_{n}\right]$ stable if

$$
\left(\forall i: \Im\left(z_{i}\right)>0\right) \Rightarrow f\left(z_{1}, \ldots, z_{n}\right) \neq 0 .
$$

For a univariate polynomial $f(z) \in \mathbb{R}[z]$ stability is equivalent to the fact that $f(z)$ has only real roots. Observe that if $f\left(z_{1}, \ldots, z_{n}\right)$ is a stable multivariate polynomial then $g(z)=f(z, \ldots, z)$ is also stable. By this observation it is clear that the following conjecture would imply the MCP conjecture:

Multivariate MCP conjecture Let $A$ be an $n \times n$ matrix with real entries weakly increasing down columns, i.e., $a_{i, j} \leq a_{i+1, j}$ for $i=1, \ldots, n-1, j=1, \ldots, n$. Then, the multivariate multi-affine polynomial $f\left(z_{1}, \ldots, z_{n}\right)=\operatorname{per}(B)$ with $b_{i, j}=a_{i, j}+z_{j}$ is stable.

In fact, the authors conjecture a stronger version of the above conjecture.

Multivariate $k$-permanent MCP conjecture Let $A$ be an $n \times m$ matrix with real entries weakly increasing down columns, i.e., $a_{i, j} \leq a_{i+1, j}$ for $i=1, \ldots, n-1, j=1, \ldots, m$. Let $B$ be the $n \times m$ matrix with entries $b_{i, j}=a_{i, j}+z_{j}$. Let $I, J$ be index sets of size $k \leq \min (n, m)$ and denote by $[B]_{I, J}$ the $k \times k$ submatrix of $B$ containing the rows $I=\left\{i_{1}, \ldots, i_{k}\right\}$ and columns $J=\left\{j_{1}, \ldots, j_{k}\right\}$. Then, the multivariate multi-affine polynomial $f\left(z_{1}, \ldots, z_{m}\right)=\sum_{I, J} \operatorname{per}\left([B]_{I, J}\right)$ is stable. The sum goes over all possible $I$ and $J, k$-subsets of $\{1, \ldots, n\}$ and $\{1, \ldots, m\}$, respectively.

\subsection{Brändén's criterion}

In our discussion we will deal with a restricted class of multivariate polynomials:

Multi-affine polynomial A multivariate polynomial is multi-affine if it has degree at most one in each variable.

Recently, in (Brä07) the following useful characterization of multi-affine stable polynomials was shown. 
Theorem 1.1 (Brändén) A multivariate multi-affine polynomial with real coefficients $f \in \mathbb{R}\left[z_{1}, \ldots, z_{n}\right]$ is stable, if and only if for all $\xi \in \mathbb{R}^{n}$ and $1 \leq i<j \leq n$

$$
\Delta_{i, j} f:=\frac{\partial f}{\partial z_{i}}(\xi) \cdot \frac{\partial f}{\partial z_{j}}(\xi)-\frac{\partial^{2} f}{\partial z_{i} \partial z_{j}}(\xi) \cdot f(\xi) \geq 0
$$

This equivalent condition is often referred to as the strong Rayleigh property.

The $k$-permanent conjecture is trivial for $k=1$. Consider the $n=k=2$ case. Let $A=\left(\begin{array}{ll}a & c \\ b & d\end{array}\right)$ denote a monotone column matrix. Then for

$$
f\left(z_{1}, z_{2}\right)=\operatorname{per}\left(\begin{array}{cc}
z_{1}+a & z_{2}+c \\
z_{1}+b & z_{2}+d
\end{array}\right)=\left(z_{1}+a\right)\left(z_{2}+d\right)+\left(z_{1}+b\right)\left(z_{2}+c\right)
$$

using Brändén's criterion we need to show that

$$
\begin{gathered}
\Delta_{1,2} f=\left(2 z_{1}+a+b\right)\left(2 z_{2}+c+d\right)-2\left(\left(z_{1}+a\right)\left(z_{2}+d\right)+\left(z_{1}+b\right)\left(z_{2}+c\right)\right)= \\
=(a+c)(b+d)-2(a d+b c)=(a-b)(c-d) \geq 0,
\end{gathered}
$$

which is a consequence of the monotone column property of $A$.

Note that it is possible to apply this method straightforwardly to the $n=k=3$ case and perhaps larger matrices, however the computations become soon intractable. In the following section we present observations that allow us to restrict ourselves to $0-1$ matrices.

\section{Reducing the conjecture to 0-1 matrices}

Lemma 2.1 If there is a counterexample to the k-permanent MCP conjecture, then there is a counterexample $A$ such that there are only two different entries in each column of $A$.

Proof: If there are no counterexamples to the conjecture the lemma is true. Otherwise, let $k$ denote the smallest number for which the $k$-permanent MCP conjecture is false. Clearly, $k>1$. Let $A$ be a minimal size counterexample for this $k$ with $n$ rows and $m$ columns, and assume that $A$ has a column with at least three different values in it. W.l.o.g., we can assume that this is the first column, i.e., $\alpha=a_{k-1,1}<a_{k, 1}=$ $\beta=a_{\ell, 1}<a_{\ell+1,1}=\gamma$ for some $1<k \leq l<n$. We will show that by changing all occurrences of $\beta$ to $\alpha$ or $\gamma$ we obtain a matrix which is also a counterexample. Clearly, the first column of the matrix will have one less different values (and all other columns remain unchanged). Hence, by repeating this procedure in each column we will arrive at a counterexample matrix that has only two different entries per column.

Using the notation of the conjecture, denote the matrix with entries $b_{i, j}=a_{i, j}+z_{j}$ by $B$ and the multivariate multi-affine polynomial obtained by summing over all $k$-permanental minors of $B$ by $f$. Since $A$ is a counterexample there are complex numbers $\xi_{i}, i=1 \ldots, m$ with positive imaginary part such that $f\left(\xi_{1}, \ldots, \xi_{m}\right)=0$. On the other hand, by expanding the permanent along the first column we get that

$$
f\left(z_{1}, \xi_{2}, \ldots, \xi_{m}\right)=z_{1} p\left(\xi_{2}, \ldots, \xi_{m}\right)+\beta q\left(\xi_{2}, \ldots, \xi_{m}\right)+r\left(\xi_{2}, \ldots, \xi_{m}\right)
$$

where the polynomials $p\left(z_{2}, \ldots, z_{m}\right), q\left(z_{2}, \ldots, z_{m}\right)$ and $r\left(z_{2}, \ldots, z_{m}\right)$ do not depend on $z_{1}$ nor on $\beta$. Note that $p$ is the polynomial obtained by summing over all $k-1$-permanental polynomial of a monotone 
matrix (obtained from $A$ by deleting the first column). And since $\Im\left(\xi_{i}\right)>0$ for $i=2 \ldots m$, by the minimality of $A$ and $k$ we get that $p\left(\xi_{2}, \ldots, \xi_{m}\right) \neq 0$.

We need to show that if we change all occurrences of $\beta$ to $\alpha$ (or all occurrences of $\beta$ to $\gamma$ ) in the column then the modified matrix is also a counterexample, i.e., $z_{1} p+\alpha q+r=0$ (or $z_{1} p+\gamma q+r=0$ ) for some $z_{1}$ with positive imaginary part. Let $w_{1}=-\frac{\alpha q+r}{p}$ and $w_{2}=-\frac{\gamma q+r}{p}$. Since $z_{1}$ is a linear function of $\beta$ in $z_{1} p+\beta q+r=0$, and $\Im\left(\xi_{1}\right)>0$ where $\xi_{1}=-\frac{\beta q+r}{p}$, it must be the case that $\Im\left(w_{1}\right)>0$ or $\Im\left(w_{2}\right)>0$.

Lemma 2.2 If there is a counterexample to the $k$-permanent MCP conjecture, then there is a counterexample $A$ with entries 0 and 1 only.

Proof: By the previous lemma we can assume that each column $j$ in $A$ has at most two different entries. Consider the case when there are two different values in each column, namely $c_{j}<d_{j}$. Since multiplying a complex number $z$ by a positive real number and adding a real number to $z$ does not change the sign of its imaginary part, $\Im(z)$, it is easy to see that $f\left(z_{1}, \ldots, z_{m}\right)=\operatorname{per}\left(a_{i j}+z_{j}\right)$ is stable if and only if $g\left(z_{1}, \ldots, z_{m}\right)=f\left(\left(d_{1}-c_{1}\right) z_{1}-c_{1}, \ldots,\left(d_{n}-c_{n}\right) z_{m}-c_{m}\right)$ is stable. To conclude, note that $g\left(z_{1}, \ldots, z_{m}\right)=\operatorname{per}\left(\tilde{a}_{i j}+z_{j}\right)$ where $\tilde{a}_{i j} \in\{0,1\}$ for all $i, j$. For the case when $c_{i}=d_{i}$ for some $i$ the proof is similar.

\section{Results for the MCP conjecture}

Note that in Lemma 2.1 the same proof goes through if we only consider matrices $A$ of size $n \times m$ with $m \leq n$, and restrict ourselves to the $k=m$ case. Then the $p \neq 0$ assumption for the coefficient of $z_{1}$ still holds, because $p$ is in fact the sum of the $(m-1)$-permanents of a matrix of size $n \times(m-1)$.

\subsection{A new proof for the $3 \times 3$ case}

By Lemmas 2.1 and 2.2, in the $3 \times 3$ case we only need to verify the monotone column $0-1$ matrices. Due to symmetry considerations we can restrict ourselves to $\left(\begin{array}{l}6 \\ 3\end{array}\right)=20$ matrices, the number of Ferrers boards fitting in a $3 \times 3$ square. Furthermore, 16 out of these matrices have an all 0 or all 1 column, which means that we can factor $z_{j}$ or $z_{j}+1$, respectively, from the permanent and reduce the question to a $3 \times 2$ matrix. Let us check the conjecture for these matrices first.

In the $3 \times 2$ case if we have an all 0 (or all 1) column the problem is trivial since we can factor the polynomial, hence it is stable. There are 3 matrices which do not have an all 0 or all 1 column:

$$
\left(\begin{array}{ll}
0 & 0 \\
0 & 0 \\
1 & 1
\end{array}\right),\left(\begin{array}{ll}
0 & 0 \\
0 & 1 \\
1 & 1
\end{array}\right) \text {, and }\left(\begin{array}{ll}
0 & 0 \\
1 & 1 \\
1 & 1
\end{array}\right) \text {. }
$$

Denote by $f_{\left(c_{1}, \ldots, c_{m}\right)}$ the polynomial obtained by taking the permanent of matrix $B$ with $b_{i j}=a_{i j}+z_{j}$, where the corresponding matrix $A$ is an $n \times m$ Ferrers matrix with $c_{j}$ ones in column $j$ for all $j$.

The permanents

$$
\begin{aligned}
& f_{(1,1)}=z_{1}\left(2 z_{2}+1\right)+z_{1}\left(2 z_{2}+1\right)+\left(z_{1}+1\right) 2 z_{2}=6 z_{1} z_{2}+2 z_{1}+2 z_{2} \\
& f_{(1,2)}=z_{1}\left(2 z_{2}+2\right)+z_{1}\left(2 z_{2}+1\right)+\left(z_{1}+1\right)\left(2 z_{2}+1\right)=6 z_{1} z_{2}+4 z_{1}+2 z_{2}+1 \\
& f_{(2,2)}=z_{1}\left(2 z_{2}+2\right)+\left(z_{1}+1\right)\left(2 z_{2}+1\right)+\left(z_{1}+1\right)\left(2 z_{2}+1\right)=6 z_{1} z_{2}+4 z_{1}+4 z_{2}+2
\end{aligned}
$$


all satisfy [1, i.e., they all have the strong Rayleigh property:

$$
\begin{aligned}
& \Delta_{1,2} f_{(1,1)}=\left(6 z_{2}+2\right)\left(6 z_{1}+2\right)-6\left(6 z_{1} z_{2}+2 z_{1}+2 z_{2}\right)=4 \geq 0, \\
& \Delta_{1,2} f_{(1,2)}=\left(6 z_{2}+4\right)\left(6 z_{1}+2\right)-6\left(6 z_{1} z_{2}+4 z_{1}+2 z_{2}+1\right)=2 \geq 0, \\
& \Delta_{1,2} f_{(2,2)}=\left(6 z_{2}+4\right)\left(6 z_{1}+4\right)-6\left(6 z_{1} z_{2}+4 z_{1}+4 z_{2}+2\right)=4 \geq 0 .
\end{aligned}
$$

Now we only need to check the following $3 \times 3$ matrices:

$$
\left(\begin{array}{lll}
0 & 0 & 0 \\
0 & 0 & 0 \\
1 & 1 & 1
\end{array}\right),\left(\begin{array}{lll}
0 & 0 & 0 \\
0 & 0 & 1 \\
1 & 1 & 1
\end{array}\right),\left(\begin{array}{lll}
0 & 0 & 0 \\
0 & 1 & 1 \\
1 & 1 & 1
\end{array}\right),\left(\begin{array}{lll}
0 & 0 & 0 \\
1 & 1 & 1 \\
1 & 1 & 1
\end{array}\right) .
$$

Following the notation from above, the permanents

$$
\begin{aligned}
& f_{(1,1,1)}=6 z_{1} z_{2} z_{3}+2 z_{1} z_{2}+2 z_{1} z_{3}+2 z_{2} z_{3} \\
& f_{(1,1,2)}=6 z_{1} z_{2} z_{3}+4 z_{1} z_{2}+2 z_{1} z_{3}+2 z_{2} z_{3}+z_{1}+z_{2} \\
& f_{(1,2,2)}=6 z_{1} z_{2} z_{3}+4 z_{1} z_{2}+4 z_{1} z_{3}+2 z_{2} z_{3}+2 z_{1}+z_{2}+z_{3} \\
& f_{(2,2,2)}=6 z_{1} z_{2} z_{3}+4 z_{1} z_{2}+4 z_{1} z_{3}+4 z_{2} z_{3}+2 z_{1}+2 z_{2}+2 z_{3}
\end{aligned}
$$

all have the strong Rayleigh property

$$
\begin{aligned}
& \Delta_{1,2} f_{(1,1,1)}=4 z_{3}^{2} \geq 0 \\
& \Delta_{1,2} f_{(1,1,2)}=\left(2 z_{3}+1\right)^{2} \geq 0 \\
& \Delta_{1,3} f_{(1,1,2)}=2 z_{2}^{2} \geq 0 \\
& \Delta_{1,2} f_{(1,2,2)}=2\left(z_{3}+1\right)^{2} \geq 0 \\
& \Delta_{2,3} f_{(1,2,2)}=\left(2 z_{1}+1\right)^{2} \geq 0 \\
& \Delta_{1,2} f_{(2,2,2)}=4\left(z_{3}+1\right)^{2} \geq 0 .
\end{aligned}
$$

Note we did not check $\Delta_{i, j} f \geq 0$ for all possible $i, j$ pairs, the remaining cases follow by symmetry.

As a consequence we obtain that the MCP conjecture holds for $3 \times 3$ matrices.

\subsection{A proof for the $4 \times 4$ case}

The above computation reveals one weakness of this method, namely that we have to check the strong Rayleigh property for all pairs, i.e., we need to verify $\Delta_{i, j} f \geq 0$ for all $i<j$. In (WW09) a new criterion was introduced to reduce the computation required.

Theorem 3.1 (Wagner and Wei) Let $f\left(z_{1}, \ldots, z_{n}\right)$ be a multi-affine polynomial with positive coefficients. Then $f$ has the strong Rayleigh property if and only if

$$
\frac{\partial f}{\partial z_{\ell}} \text { and } f_{\mid z_{\ell}=0}=f\left(z_{1}, \ldots, z_{\ell-1}, 0, z_{\ell+1}, \ldots, z_{n}\right)
$$

have the strong Rayleigh property for all $\ell$, and (1) holds for some pair $i<j$. 
This theorem is helpful because it is sufficient to check now $\Delta_{i, j} f \geq 0$ for only one index pair. We were checking the stability of $\partial f / \partial z_{\ell}$ already. Since, if $A$ is an $n \times n$ matrix then $\partial f / \partial z_{\ell}$ is the permanent corresponding to the $n \times(n-1)$ matrix obtained by removing column $\ell$ from $A$ (we were checking this, in case column $\ell$ had only zeros or only ones). Checking the stability of $f_{\mid z_{\ell}=0}$ is an extra overhead but overall saves more time than if we had to check $\Delta_{i, j} f \geq 0$ for all index pairs.

Let us verify the conjecture for $4 \times 4$ matrices. Again, to verify the conjecture for matrices with an all 0 or all 1 columns we reduce the problem to $4 \times 3$ and $4 \times 2$ matrices consequently. The permanents of $4 \times 1$ matrices are trivially stable. Here are the 6 matrices of size $4 \times 2$ with no all 0 or all 1 columns:

$$
\left(\begin{array}{ll}
0 & 0 \\
0 & 0 \\
0 & 0 \\
1 & 1
\end{array}\right),\left(\begin{array}{ll}
0 & 0 \\
0 & 0 \\
0 & 1 \\
1 & 1
\end{array}\right),\left(\begin{array}{ll}
0 & 0 \\
0 & 1 \\
0 & 1 \\
1 & 1
\end{array}\right),\left(\begin{array}{ll}
0 & 0 \\
0 & 0 \\
1 & 1 \\
1 & 1
\end{array}\right),\left(\begin{array}{ll}
0 & 0 \\
0 & 1 \\
1 & 1 \\
1 & 1
\end{array}\right),\left(\begin{array}{ll}
0 & 0 \\
1 & 1 \\
1 & 1 \\
1 & 1
\end{array}\right) .
$$

We need to verify the strong Rayleigh property for the corresponding polynomials:

$$
\begin{aligned}
& f_{(1,1)}=12 z_{1} z_{2}+3 z_{1}+3 z_{2} \\
& f_{(1,2)}=12 z_{1} z_{2}+6 z_{1}+3 z_{2}+1 \\
& f_{(1,3)}=12 z_{1} z_{2}+9 z_{1}+3 z_{2}+2 \\
& f_{(2,2)}=12 z_{1} z_{2}+6 z_{1}+6 z_{2}+2 \\
& f_{(2,3)}=12 z_{1} z_{2}+9 z_{1}+6 z_{2}+4 \\
& f_{(3,3)}=12 z_{1} z_{2}+9 z_{1}+9 z_{2}+6
\end{aligned}
$$

They are all stable since,

$$
\begin{array}{cc}
\Delta_{1,2} f_{(1,1)}=\left(12 z_{1}+3\right)\left(12 z_{2}+3\right)-12\left(12 z_{1} z_{2}+3 z_{1}+3 z_{2}\right) & =9 \geq 0 \\
\Delta_{1,2} f_{(1,2)}=\left(12 z_{1}+3\right)\left(12 z_{2}+6\right)-12\left(12 z_{1} z_{2}+6 z_{1}+3 z_{2}+1\right) & =6 \geq 0 \\
\Delta_{1,2} f_{(1,3)}=\left(12 z_{1}+3\right)\left(12 z_{2}+9\right)-12\left(12 z_{1} z_{2}+9 z_{1}+3 z_{2}+2\right) & =3 \geq 0 \\
\Delta_{1,2} f_{(2,2)}=\left(12 z_{1}+6\right)\left(12 z_{2}+6\right)-12\left(12 z_{1} z_{2}+6 z_{1}+6 z_{2}+2\right) & =12 \geq 0 \\
\Delta_{1,2} f_{(2,3)}=\left(12 z_{1}+6\right)\left(12 z_{2}+9\right)-12\left(12 z_{1} z_{2}+9 z_{1}+6 z_{2}+4\right) & =6 \geq 0 \\
\Delta_{1,2} f_{(3,3)}=\left(12 z_{1}+9\right)\left(12 z_{2}+9\right)-12\left(12 z_{1} z_{2}+9 z_{1}+9 z_{2}+6\right) & =9 \geq 0 .
\end{array}
$$

Now, we need to verify the stability of the $4 \times 3$ matrices (with no all 0 or all 1 columns):

$$
\begin{aligned}
& \left(\begin{array}{lll}
0 & 0 & 0 \\
0 & 0 & 0 \\
0 & 0 & 0 \\
1 & 1 & 1
\end{array}\right),\left(\begin{array}{lll}
0 & 0 & 0 \\
0 & 0 & 0 \\
0 & 0 & 1 \\
1 & 1 & 1
\end{array}\right),\left(\begin{array}{lll}
0 & 0 & 0 \\
0 & 0 & 1 \\
0 & 0 & 1 \\
1 & 1 & 1
\end{array}\right),\left(\begin{array}{lll}
0 & 0 & 0 \\
0 & 0 & 0 \\
0 & 1 & 1 \\
1 & 1 & 1
\end{array}\right),\left(\begin{array}{lll}
0 & 0 & 0 \\
0 & 0 & 1 \\
0 & 1 & 1 \\
1 & 1 & 1
\end{array}\right), \\
& \left(\begin{array}{lll}
0 & 0 & 0 \\
0 & 1 & 1 \\
0 & 1 & 1 \\
1 & 1 & 1
\end{array}\right),\left(\begin{array}{lll}
0 & 0 & 0 \\
0 & 0 & 0 \\
1 & 1 & 1 \\
1 & 1 & 1
\end{array}\right),\left(\begin{array}{lll}
0 & 0 & 0 \\
0 & 0 & 1 \\
1 & 1 & 1 \\
1 & 1 & 1
\end{array}\right),\left(\begin{array}{lll}
0 & 0 & 0 \\
0 & 1 & 1 \\
1 & 1 & 1 \\
1 & 1 & 1
\end{array}\right),\left(\begin{array}{lll}
0 & 0 & 0 \\
1 & 1 & 1 \\
1 & 1 & 1 \\
1 & 1 & 1
\end{array}\right) .
\end{aligned}
$$


The polynomials and their Rayleigh differences are:

$$
\begin{aligned}
& f_{(1,1,1)}=24 z_{1} z_{2} z_{3}+6 z_{1} z_{2}+6 z_{1} z_{3}+6 z_{2} z_{3} \\
& f_{(1,1,2)}=24 z_{1} z_{2} z_{3}+12 z_{1} z_{2}+6 z_{1} z_{3}+6 z_{2} z_{3}+2 z_{1}+2 z_{2} \\
& f_{(1,1,3)}=24 z_{1} z_{2} z_{3}+18 z_{1} z_{2}+6 z_{1} z_{3}+6 z_{2} z_{3}+4 z_{1}+4 z_{2} \\
& f_{(1,2,2)}=24 z_{1} z_{2} z_{3}+12 z_{1} z_{3}+12 z_{1} z_{2}+6 z_{2} z_{3}+4 z_{1}+2 z_{2}+2 z_{3} \\
& f_{(1,2,3)}=24 z_{1} z_{2} z_{3}+18 z_{1} z_{2}+12 z_{1} z_{3}+6 z_{2} z_{3}+8 z_{1}+4 z_{2}+2 z_{3}+1 \\
& f_{(1,3,3)}=24 z_{1} z_{2} z_{3}+18 z_{1} z_{2}+18 z_{1} z_{3}+6 z_{2} z_{3}+12 z_{1}+4 z_{2}+4 z_{3}+2 \\
& f_{(2,2,2)}=24 z_{1} z_{2} z_{3}+12 z_{1} z_{2}+12 z_{1} z_{3}+12 z_{2} z_{3}+4 z_{1}+4 z_{2}+4 z_{3} \\
& f_{(2,2,3)}=24 z_{1} z_{2} z_{3}+18 z_{1} z_{2}+12 z_{1} z_{3}+12 z_{2} z_{3}+8 z_{1}+8 z_{2}+4 z_{3}+2 \\
& f_{(2,3,3)}=24 z_{1} z_{2} z_{3}+18 z_{1} z_{2}+18 z_{1} z_{3}+12 z_{2} z_{3}+12 z_{1}+8 z_{2}+8 z_{3}+4 \\
& f_{(3,3,3)}=24 z_{1} z_{2} z_{3}+18 z_{1} z_{2}+18 z_{1} z_{3}+18 z_{2} z_{3}+12 z_{1}+12 z_{2}+12 z_{3}+6 \\
& \Delta_{1,2} f_{(1,1,1)}=36 z_{3}^{2} \\
& \Delta_{1,2} f_{(1,1,2)}=4\left(3 z_{3}+1\right)^{2} \\
& \Delta_{1,3} f_{(1,1,2)}=24 z_{2}^{2} \\
& \Delta_{1,2} f_{(1,1,3)}=4\left(3 z_{3}+2\right)^{2} \\
& \Delta_{1,3} f_{(1,1,3)}=12 z_{2}^{2} \\
& \Delta_{1,2} f_{(1,2,2)}=8\left(3 z_{3}^{2}+3 z_{3}+1\right) \\
& \Delta_{2,3} f_{(1,2,2)}=4\left(12 z_{1}^{2}+6 z_{1}+1\right) \\
& \Delta_{1,2} f_{(1,2,3)}=2\left(12 z_{3}^{2}+18 z_{3}+7\right) \\
& \Delta_{1,3} f_{(1,2,3)}=4\left(3 z_{2}^{2}+3 z_{2}+1\right) \\
& \Delta_{2,3} f_{(1,2,3)}=2\left(12 z_{1}^{2}+6 z_{1}+1\right) \\
& \Delta_{1,2} f_{(1,3,3)}=12\left(z_{3}+1\right)^{2} \\
& \Delta_{2,3} f_{(1,3,3)}=4\left(3 z_{1}+1\right)^{2} \\
& \Delta_{1,2} f_{(2,2,2)}=16\left(3 z_{3}^{2}+3 z_{3}+1\right) \\
& \Delta_{1,2} f_{(2,2,3)}=4\left(12 z_{3}^{2}+18 z_{3}+7\right) \\
& \Delta_{1,3} f_{(2,2,3)}=8\left(3 z_{2}^{2}+3 z_{2}+1\right) \\
& \Delta_{1,2} f_{(2,3,3)}=24\left(z_{3}+1\right)^{2} \\
& \Delta_{2,3} f_{(2,3,3)}=4\left(3 z_{1}+2\right)^{2} \\
& \Delta_{1,2} f_{(3,3,3)}=36\left(z_{3}+1\right)^{2}
\end{aligned}
$$

Finally, we have to show the stability of $4 \times 4$ matrices. Instead of computing the Rayleigh differences potentially $\left(\begin{array}{l}4 \\ 2\end{array}\right)=6$ times for each matrix, we employ Theorem 3.1 and compute only one Rayleigh difference per matrix. We already have that the partial derivatives are stable, since these are exactly the polynomials which are the permanents of $4 \times 3$ matrices. Now we need to show the stability of $f_{\mid z_{\ell}=0}$. 
These polynomials have one of the three following forms. They can be a permanent of a $3 \times 3$ matrix obtained by removing the last row of the $4 \times 3$ matrix, or the sum of two permanents (one obtained by removing the third row and another obtained by removing the last row of the original matrix), or three permanents of $3 \times 3$ matrices (obtained by the respective submatrices of the given $4 \times 3$ matrix by removing the second, third and last row).

The $3 \times 3$ case we have already solved in Section 3.1, the sum of two permanents can be reduced to a single $3 \times 3$ permanent case, by expanding the permanent along the last row:

$$
\begin{aligned}
\operatorname{per}\left(\begin{array}{cccc}
z_{1}+a_{1} & z_{2}+a_{2} & z_{3}+a_{3} & 0 \\
z_{1}+b_{1} & z_{2}+b_{2} & z_{3}+b_{3} & 0 \\
z_{1}+c_{1} & z_{2}+c_{2} & z_{3}+c_{3} & 1 \\
z_{1}+d_{1} & z_{2}+d_{2} & z_{3}+d_{3} & 1
\end{array}\right)= & \operatorname{per}\left(\begin{array}{ccc}
z_{1}+a_{1} & z_{2}+a_{2} & z_{3}+a_{3} \\
z_{1}+b_{1} & z_{2}+b_{2} & z_{3}+b_{3} \\
z_{1}+c_{1} & z_{2}+c_{2} & z_{3}+c_{3}
\end{array}\right)+ \\
& \operatorname{per}\left(\begin{array}{ccc}
z_{1}+a_{1} & z_{2}+a_{2} & z_{3}+a_{3} \\
z_{1}+b_{1} & z_{2}+b_{2} & z_{3}+b_{3} \\
z_{1}+d_{1} & z_{2}+d_{2} & z_{3}+d_{3}
\end{array}\right) \\
= & 2 \operatorname{per}\left(\begin{array}{ccc}
z_{1}+a_{1} & z_{2}+a_{2} & z_{3}+a_{3} \\
z_{1}+b_{1} & z_{2}+b_{2} & z_{3}+b_{3} \\
z_{1}+\frac{c_{1}+d_{1}}{2} & z_{2}+\frac{c_{2}+d_{2}}{2} & z_{3}+\frac{c_{3}+d_{3}}{2}
\end{array}\right)
\end{aligned}
$$

Note that in this case the other rows are identical, and since the entries in the last rows are the largest ones their average also preserves the monotone column property.

For the sum of three permanents we can also argue similarly. Note that if there are two columns with the same number of ones, then two of the summands are identical and we can sum the permanents by expanding along the row in which they differ. Here factoring out 3 and placing the average in the row will preserve the monotone column property. The only case when all columns have different number of ones is when

$$
A=\left(\begin{array}{lll}
0 & 0 & 0 \\
0 & 0 & 1 \\
0 & 1 & 1 \\
1 & 1 & 1
\end{array}\right)
$$

The corresponding polynomial $f=18 z_{1} z_{2} z_{3}+12 z_{1} z_{2}+8 z_{1} z_{3}+4 z_{2} z_{3}+4 z_{1}+2 z_{2}+z_{3}$ is stable, because the

$$
\begin{aligned}
\Delta_{1,2} f & =2\left(7 z_{3}^{2}+10 z_{3}+4\right) \\
\Delta_{1,3} f & =4\left(3 z_{2}^{2}+3 z_{2}+1\right) \\
\Delta_{2,3} f & =2\left(12 z_{1}^{2}+6 z_{1}+1\right)
\end{aligned}
$$

differences are always positive. 
We check the strong Rayleigh property for all the 15 matrices of size $4 \times 4$ with no all 0 or all 1 columns:

$$
\begin{aligned}
& \Delta_{1,2} f_{(1,1,1,1)}=36 z_{3}^{2} z_{4}^{2} \\
& \Delta_{1,2} f_{(1,1,1,2)}=4 z_{3}^{2}\left(3 z_{4}+1\right)^{2} \\
& \Delta_{1,2} f_{(1,1,1,3)}=4 z_{3}^{2}\left(3 z_{4}+2\right)^{2} \\
& \Delta_{1,2} f_{(1,1,2,2)}=4\left(3 z_{3} z_{4}+z_{3}+z_{4}\right)^{2} \\
& \Delta_{1,2} f_{(1,1,2,3)}=\left(6 z_{3} z_{4}+4 z_{3}+3 z_{4}+1\right)^{2} \\
& \Delta_{1,2} f_{(1,1,3,3)}=4\left(3 z_{3} z_{4}+2 z_{3}+2 z_{4}+1\right)^{2} \\
& \Delta_{1,2} f_{(1,2,2,2)}=8 z_{3} z_{4}+24 z_{3}^{2} z_{4}^{2}+8 z_{3}^{2}+8 z_{4}^{2}+24 z_{3}^{2} z_{4}+24 z_{3} z_{4}^{2} \\
& \Delta_{1,2} f_{(1,2,2,3)}=2+8 z_{4}^{2}+8 z_{3}+8 z_{4}+36 z_{3}^{2} z_{4}+24 z_{3} z_{4}^{2}+14 z_{3}^{2}+24 z_{3}^{2} z_{4}^{2}+28 z_{3} z_{4} \\
& \Delta_{1,2} f_{(1,2,3,3)}=8+14 z_{4}^{2}+20 z_{3}+20 z_{4}+36 z_{3}^{2} z_{4}+36 z_{3} z_{4}^{2}+14 z_{3}^{2}+24 z_{3}^{2} z_{4}^{2}+52 z_{3} z_{4} \\
& \Delta_{1,2} f_{(1,3,3,3)}=12\left(z_{3}+1\right)^{2}\left(z_{4}+1\right)^{2} \\
& \Delta_{1,2} f_{(2,2,2,2)}=16 z_{4}^{2}+48 z_{3}^{2} z_{4}+48 z_{3} z_{4}^{2}+16 z_{3}^{2}+48 z_{3}^{2} z_{4}^{2}+16 z_{3} z_{4} \\
& \Delta_{1,2} f_{(2,2,2,3)}=4+16 z_{4}^{2}+16 z_{3}+16 z_{4}+72 z_{3}^{2} z_{4}+48 z_{3} z_{4}^{2}+28 z_{3}^{2}+48 z_{3}^{2} z_{4}^{2}+56 z_{3} z_{4} \\
& \Delta_{1,2} f_{(2,2,3,3)}=16+28 z_{4}^{2}+40 z_{3}+40 z_{4}+72 z_{3}^{2} z_{4}+72 z_{3} z_{4}^{2}+28 z_{3}^{2}+48 z_{3}^{2} z_{4}^{2}+104 z_{3} z_{4} \\
& \Delta_{1,2} f_{(2,3,3,3)}=24\left(z_{3}+1\right)^{2}\left(z_{4}+1\right)^{2} \\
& \Delta_{1,2} f_{(3,3,3,3)}=36\left(z_{4}+1\right)^{2}\left(z_{3}+1\right)^{2}
\end{aligned}
$$

The 6 differences which are not complete squares are also non-negative, since they have the following non-positive discriminants (when they are considered as a polynomial in $z_{3}$ ):

$-192\left(z_{4}+1\right)^{2} z_{4}^{2},-48\left(z_{4}+1\right)^{2}\left(2 z_{4}+1\right)^{2},-48\left(z_{4}+1\right)^{4},-768\left(z_{4}+1\right)^{2} z_{4}^{2},-192\left(z_{4}+1\right)^{2}\left(2 z_{4}+1\right)^{2},-192\left(z_{4}+1\right)^{4}$

and positive leading coefficients:

$8\left(3 z_{4}^{2}+3 z_{4}+1\right), 2\left(12 z_{4}^{2}+18 z_{4}+7\right), 2\left(12 z_{4}^{2}+18 z_{4}+7\right), 16\left(3 z_{4}^{2}+3 z_{4}+1\right), 4\left(12 z_{4}^{2}+18 z_{4}+7\right), 4\left(12 z_{4}^{2}+18 z_{4}+7\right)$.

\subsection{Proof for a $5 \times 5$ matrix and the Eulerian polynomials}

There is an interesting connection between the Eulerian polynomial and the multivariate MCP conjecture. Let $A$ be the $n \times n$ matrix with all zeros above and on the diagonal and all ones below. Denote the permanent of $B$ where $b_{i j}=a_{i j}+z_{j}$ by $f\left(z_{1}, \ldots, z_{n}\right)$. The univariate polynomial obtained by setting $z_{i}=z$ for all $i$ is the Eulerian polynomial modified by a rational change of variables:

$$
f(z, \ldots, z)=(z+1)^{n} A_{n}\left(\frac{z}{1+z}\right)=\sum_{k=1}^{n} A(n, k) z^{k}(1+z)^{n-k},
$$

where $A_{n}(z)$ is the generating polynomial of the Eulerian numbers, $A(n, k), e . g$., the number of permutations of $n$ letters with $k$ weak excedances. (A number $i$ is a weak excedance in permutation $\pi=$ $\pi_{1} \pi_{2} \cdots \pi_{n}$ if $\pi_{i} \geq i$.) To show (2) we can identify permutations of $n$ letters with placements of $n$ rooks on an $n \times n$ board, with a rook on $(i, j)$ interpreted as $\pi_{i}=j$ in the permutation $\pi=\pi_{1} \pi_{2} \cdots \pi_{n}$. The 
$z^{k}(1+z)^{n-k}$ terms in the expansion of the permanent $f(z, \ldots, z)$ correspond to rook placements with $k$ rooks on or above the diagonal (each rook contributes a a factor of $z$ to the term). Hence the coefficient of $z^{k}(1+z)^{n-k}$ is exactly $A(n, k)$.

Therefore, this multivariate generalization of the Eulerian polynomials is a natural candidate to be proven stable, which would imply the well-known fact that Eulerian polynomials have only real zeros.

We already proved that for $n \leq 4$ these polynomials are stable. Now we show that for $n=5$ this special case of the multivariate MCP conjecture also holds.

Let

$$
A=\left(\begin{array}{lllll}
0 & 0 & 0 & 0 & 0 \\
0 & 0 & 0 & 0 & 1 \\
0 & 0 & 0 & 1 & 1 \\
0 & 0 & 1 & 1 & 1 \\
0 & 1 & 1 & 1 & 1
\end{array}\right) .
$$

Let $f=f_{(0,1,2,3,4)}$. We check the strong Rayleigh property for all $1 \leq i<j \leq 5$. Since we can factor out $z_{1}$ from $f$ note that $\Delta_{1,2} f=\Delta_{1,3} f=\Delta_{1,4} f=\Delta_{1,5} f=0$.

$$
\begin{aligned}
\Delta_{2,3} f & =2 z_{1}^{2}\left(216 z_{4}^{2} z_{5}^{2}+336 z_{4}^{2} z_{5}+240 z_{4} z_{5}^{2}+354 z_{4} z_{5}+132 z_{4}^{2}+132 z_{4}+72 z_{5}^{2}+102 z_{5}+37\right) \\
\Delta_{2,4} f & =4 z_{1}^{2}\left(75 z_{3} z_{5}+120 z_{3}^{2} z_{5}+48 z_{3} z_{5}^{2}+72 z_{3}^{2} z_{5}^{2}+12 z_{5}^{2}+18 z_{5}+7+51 z_{3}^{2}+30 z_{3}\right) \\
\Delta_{2,5} f & =4 z_{1}^{2}\left(27 z_{3} z_{4}+48 z_{3}^{2} z_{4}+24 z_{3} z_{4}^{2}+36 z_{3}^{2} z_{4}^{2}+6 z_{4}^{2}+6 z_{4}+2+18 z_{3}^{2}+9 z_{3}\right) \\
\Delta_{3,4} f & =2 z_{1}^{2}\left(150 z_{2} z_{5}+96 z_{5}^{2} z_{2}+288 z_{2}^{2} z_{5}^{2}+480 z_{2}^{2} z_{5}+12 z_{5}^{2}+18 z_{5}+7+60 z_{2}+204 z_{2}^{2}\right) \\
\Delta_{3,5} f & =4 z_{1}^{2}\left(27 z_{2} z_{4}+72 z_{2}^{2} z_{4}^{2}+24 z_{4}^{2} z_{2}+3 z_{4}^{2}+3 z_{4}+1+96 z_{2}^{2} z_{4}+9 z_{2}+36 z_{2}^{2}\right) \\
\Delta_{4,5} f & =2 z_{1}^{2}\left(216 z_{2}^{2} z_{3}^{2}+66 z_{2} z_{3}+96 z_{3}^{2} z_{2}+1+192 z_{2}^{2} z_{3}+12 z_{2}+12 z_{3}^{2}+6 z_{3}+48 z_{2}^{2}\right)
\end{aligned}
$$

We verified by a computer that these are always non-negative by the following procedure. For example, consider $g\left(z_{2}, z_{3}\right)=\Delta_{4,5} f / 2 z_{1}^{2}$. The coefficient of $z_{3}^{2}$ in $g\left(z_{2}, z_{3}\right)$ is $12\left(18 z^{2}+8 z_{2}+1\right)>0$ and the discriminant of $g\left(z_{2}, z_{3}\right)$ viewed as a polynomial in $z_{3}$ is $-12\left(384 z_{2}^{4}+288 z_{2}^{3}+93 z_{2}^{2}+14 z_{2}+1\right)<0$, since this quartic polynomial has negative leading coefficient and has no real roots.

\section{References}

[BB08] Julius Borcea and Petter Brändén. Applications of stable polynomials to mixed determinants: Johnson's conjectures, unimodality, and symmetrized Fischer products. Duke Mathematical Journal, 143(2):205-223, 2008.

[Brä07] Petter Brändén. Polynomials with the half-plane property and matroid theory. Advances in Mathematics, 216(1):302-320, December 2007.

[Hag00] James Haglund. Further investigations involving rook polynomials with only real zeros. $E u$ ropean Journal of Combinatorics, 21(8):1017-1037, 2000.

[HL72] Ole J. Heilmann and Elliott H. Lieb. Theory of monomer-dimer systems. Communications in Mathematical Physics, 25(3):190-232, 1972. 
[HOW99] James Haglund, Ken Ono, and David G. Wagner. Theorems and conjectures involving rook polynomials with only real zeros. In Topics in Number Theory, volume 467 of Mathematics and Its Applications, pages 207-222. Kluwer, 1999.

[May] Raymond A. Mayer. Personal communication.

[Sok05] Alan D. Sokal. The multivariate Tutte polynomial (alias Potts model) for graphs and matroids. In Surveys in Combinatorics, volume 327 of London Mathematical Society Lecture Note Series, pages 173-226. Cambridge University Press, 2005.

[WW09] David G. Wagner and Yehua Wei. A criterion for the half-plane property. Discrete Mathematics, 309(6):1385-1390, April 2009. 
